\title{
Erratum to: Constant scalar curvature metrics on Hirzebruch surfaces
}

\section{Nobuhiko Otoba}

Published online: 20 July 2014

C) Springer Science+Business Media Dordrecht 2014

\section{Erratum to: Ann Glob Anal Geom DOI 10.1007/s10455-014-9419-z}

The original publication unfortunately contains a typo in one equation.

The equation (32)

$$
f^{\prime \prime}=-f^{3}-\frac{R-8}{2} \quad(\text { INCORRECT })
$$

should read

$$
f^{\prime \prime}=-f^{3}-\frac{R-8}{2} f \quad(\text { CORRECT })
$$

Since the missing $f$ is taken into account in the succeeding arguments and computations, this never affects the content of the paper.

The online version of the original article can be found under doi:10.1007/s10455-014-9419-z.

N. Otoba $(\bowtie)$

Keio University, 3-14-1 Hiyoshi, Kohoku-ku, Yokohama, Kanagawa 223-8522, Japan

e-mail: otoba@math.keio.ac.jp 